a. Consultant, Hospital Nacional de Pediatría J. P. Garrahan, Autonomous City of Buenos Aires.

b. Division of Pediatric Hepatology, Hospital Municipal de Niños de San Justo, Province of Buenos Aires.

c. Division of Hepatology and Pediatric Liver Transplant, Hospital Alemán, Autonomous City of Buenos Aires.

d. Division of Pediatric Nutrition, Hospital Universitario Austral, Pilar, Province of Buenos Aires.

e. Division of Hepatology, Hospital de Pediatría J. P. Garrahan, Autonomous City of Buenos Aires.

f. Multipurpose day hospital, Hospital de Niños Ricardo Gutiérrez, Autonomous City of Buenos Aires.

g. Division for Liver, Bile Ducts, and Pancreas Care, Hospital Nacional Prof. Alejandro Posadas, El Palomar, Province of Buenos Aires.

h. Unit 4, Division of Hepatology, Hospital de Niños Ricardo Gutiérrez, Autonomous City of Buenos Aires.

i. Division of Hepatology and Liver Transplant, Liver Unit, Hospital Universitario Austral, Pilar, Province of Buenos Aires.

j. Immunological and Pathophysiological Studies Institute (Instituto de Estudios Inmunológicos y Fisiopatológicos, IIFP), Department of Biological Sciences, School of Exact Sciences, Universidad Nacional de La Plata, and National Scientific and Technical Research Council (Consejo Nacional
de Investigaciones Científicas y Técnicas, CONICET), La Plata, Province of Buenos Aires.

E-mail address:

Luisa Bay, M.D.

bay.luisa@gmail.com

Funding:

None.

Conflict of interest: The authors Luisa Bay, M.D., Federico Piñero, M.D. Eduardo Fassio, M.D., and Paula Rozenfeld, M.D., state that they receive professional fees from Alexion Pharmaceuticals for their teaching dissertations.

Received: 8-22-2016 Accepted: 11-28-2016

\title{
Liver disease and dyslipidemia as a manifestation of lysosomal acid lipase deficiency (LAL-D). Clinical and diagnostic aspects, and a new treatment. An update
}

\author{
Luisa Bay, M.D. ${ }^{a}$, Cristina Cañero Velasco, M.D. ${ }^{b}$, Mirta Ciocca, M.D. ${ }^{c}$, Andrea Cotti, M.D. ${ }^{d}$, \\ Miriam Cuarterolo, M.D. ${ }^{e}$, Alejandro Fainboim, M.D. $f$, Eduardo Fassio, M.D. ${ }^{g}$, \\ Marcela Galoppo, M.D. ${ }^{h}$, Federico Piñero, M.D. ${ }^{i}$ and Paula Rozenfeld, M.D. ${ }^{j}$
}

\begin{abstract}
Lysosomal acid lipase deficiency (LAL-D) is still a little recognized genetic disease with significant morbidity and mortality in children and adults. This document provides guidance on when to suspect LAL-D and how to diagnose it. It is recommended to add lysosomal acid lipase deficiency to the list of differential diagnoses of sepsis, oncological diseases, storage diseases, persistent diarrhea, chronic malnutrition, and hemophagocytic lymphohistiocytosis. It should also be considered in young patients with dyslipidemiaand atherosclerosisaswell as diseases associated with fatty liver and / or hepatomegaly. LAL-D should be suspected in patients with hepatomegaly, hyperlipidemia and / or elevated transaminases found during routine checks or testing for other conditions, and in patients with cryptogenic cirrhosis. At present, there is the option of a specific enzyme replacement treatment.

Key words: cirrhosis, dyslipidemias, Wolman disease, non-alcoholic fatty liver disease, lysosomal acid lipase deficiency.
\end{abstract}

http:/ / dx.doi.org/10.5546/ aap.2017.eng.287

To cite: Bay L, Cañero Velasco C, Ciocca M, et al. Liver disease and dyslipidemia as a manifestation of lysosomal acid lipase deficiency (LAL-D). Clinical and diagnostic aspects, and a new treatment. An update. Arch Argent Pediatr 2017;115(3):287-293.

\section{INTRODUCTION}

Liver disease and dyslipidemia are frequent findings in everyday practice, and common signs of lysosomal acid lipase deficiency (LAL-D), a lowprevalence storage disease. It follows an autosomal recessive inheritance pattern and is caused by mutations in the LIPA gene, located in chromosome 10q23.2-23.3. ${ }^{1}$ This enzyme is involved in the metabolism and breakdown of cholesteryl esters and triglycerides that accumulate in different tissue cells when the enzyme is reduced or absent. The most commonly affected organs and systems include the liver, spleen, adrenal glands, hematopoietic system, vascular endothelium, intestines, and lymph nodes. The clinical manifestations of LAL-D vary depending on age. ${ }^{2}$ Liver involvement and dyslipidemia are the prevalent clinical presentations, and for this reason some patients may be misdiagnosed.

\section{OBJECTIVE}

To review LAL-D, its clinical manifestations, differential diagnoses, diagnostic confirmation, and new therapeutic options.

\section{Clinical manifestations}

The age at the time of onset, clinical spectrum, and disease course vary widely. There is a severe infant form or Wolman disease ${ }^{4,5}$ first described in 1956, and a late onset pediatric, adolescent, or adult form known as cholesteryl ester storage disease (CESD), first described in 1963. ${ }^{2}$ The phenotype depends on the type of LIPA gene mutation and the severity of enzyme deficiency.

The severe form occurs in the first months of life, with an almost null enzyme activity (LAL) or $<1 \%$ of normal activity. ${ }^{5}$ Children appear healthy at birth but, between 1 and 2 months old, they have malaise, failure to thrive, progressive malnutrition, marked abdominal distension caused by malabsorption and 
hepatosplenomegaly, recurrent vomiting, chronic diarrhea (steatorrhea), and liver involvement with rapid development of fibrosis and/or cholestasis and / or liver failure. Elevated transaminases (50-95\% of cases) and hypersplenism (anemia/ thrombocytopenia) are also observed. ${ }^{2}$ Approximately $50 \%$ of patients have bilateral calcifications of the adrenal glands. ${ }^{6,7}$

Cholesterol levels are elevated or normal with reduced high-density lipoproteins (HDL) and increased very low-density lipoproteins (VLDL); triglycerides may be normal or elevated. ${ }^{8}$ Developmental delay and / or irritability are secondary to malnutrition, overall compromise, and hospitalization resulting from the central nervous system involvement caused by this disease. ${ }^{9}$ The natural course of this clinical form results in death in the first year of life. ${ }^{10-12}$

The subtype of LAL-D that starts during childhood, adolescence, or adulthood implies $1-12 \%$ of normal enzyme activity; lipid accumulation leads to progressive liver damage, elevated transaminases, steatosis, fibrosis and/ or cirrhosis. These patients develop chronic dyslipidemia, which leads to early atherosclerosis (infarction, aneurism, stroke). ${ }^{13-14}$

In a series of 48 patients with this subtype of LAL-D, the median age at the time of disease reporting was 5.8 years old (maximum: 46 years old); $56 \%$ were younger than 20 years old and $17 \%$, younger than 10 years old. Symptoms started before 10 years old in $75 \%$ of patients. Alanine aminotransferase (ALT), aspartate aminotransferase (AST), and gamma-glutamyl transferase (GGT) were elevated in $92 \%, 59 \%$, and $20 \%$ of patients, respectively. Hepatomegaly, which is less common in the early onset subtype, was reported in $54 \%$ of patients whereas splenomegaly was reported in only $23 \% .{ }^{16}$

Dyslipidemia in LAL-D is indistinguishable from that occurring in other more common etiologies. Total cholesterol, LDL-C, VLDL-C, and triglycerides are elevated, and HDL-C is reduced. ${ }^{1,4,5}$ Pediatric patients have shown LDL-C $\geq 130 \mathrm{mg} / \mathrm{dL}$ and HDL-C $\leq 45 \mathrm{mg} / \mathrm{dL} \cdot{ }^{11}$ Cerebral infarction and hyperlipidemia have been reported at 13 years old, with symptomatic gallbladder dysfunction ${ }^{16}$ (Table 1).

\section{Prevalence}

Although more than 40 mutations have been described, ${ }^{17}$ the mutation in exon 8, E8SJM (c.894G $>$ A) has been identified in $50-60 \%$ of patients with the late onset subtype and of European descent. ${ }^{18}$ It was detected in approximately 1:200 heterozygous healthy German individuals. Thus, prevalence may be expected to be 1:40 000 German newborn infants, and 1:300 000 Hispanics and Caucasians. ${ }^{19,20}$ The number of reported cases has been much lower than these estimates, which suggests a non-specific clinical course and the absence of a characteristic phenotype. No studies have been conducted regarding the prevalence of LAL-D in Argentina.

\section{Diagnosis of lysosomal acid lipase deficiency at the lab}

The definitive diagnosis of LAL-D is made by demonstrating the deficiency or absence of LAL enzyme activity (EC 3.1.1.13) in isolated peripheral blood leukocytes or fibroblast cell cultures. ${ }^{21}$ Enzyme determination may also be done from blood spots dried on filter paper, ${ }^{22}$ which allows for the proper submission of lab specimens from remote locations, retrospective diagnosis, and population level screening. However, if filter paper samples are abnormal, results should be confirmed using leukocytes or fibroblasts. This test is useful to confirm the diagnosis of homozygous individuals, but not for heterozygous ones, who may have a normal enzyme activity.

The molecular genetic test consists in identifying the pathogenic mutations in both alleles of the LIPA gene, and is considered a supplementary diagnostic test. ${ }^{23}$ Mutation identification is useful to carry out family studies and, therefore, provide a diagnosis and genetic counseling to heterozygous family members.

\section{Pathophysiology}

Acid lipase acts on hepatocyte lipid metabolism, and acid lipase deficiency, as described from a pathophysiological perspective in Figure 1. A, explains dyslipidemia and neutral lipid accumulation in the lysosome, resulting in liver damage. ${ }^{24}$

\section{Differential diagnosis}

Although there are biochemical and genetic tests for the diagnosis of LAL-D, detecting this disease depends on clinical suspicion, which is low. ${ }^{11,12}$ Clinical and biochemical manifestations are consistent with other cardiovascular, liver or metabolic diseases. ${ }^{1,12}$ LAL-D should be included in the list of differential diagnoses of liver conditions and dyslipidemias. Given its severe morbidity and early mortality, patients should 
TABLE 1. Lysosomal acid lipase deficiency phenotype: from the severe form in infants (Wolman disease) to the late-onset form in children, adolescents, and adults (cholesteryl ester storage disease) (modified from Grawoski, 2014)

\begin{tabular}{lcc}
\hline Clinical signs & Newborns and infants & Children, adolescents and adults \\
\hline Age at onset & $0-3$ months old & From childhood to adulthood \\
Age at death & \pm 6 months old & Varied \\
Hepatomegaly & ++++ & $+/++$ \\
Liver fibrosis / cirrhosis & ++ & $+/+++$ \\
Splenomegaly & +++ & $+/-$ \\
Malabsorption & +++ & $+/-$ \\
Emaciation & +++ & \pm \\
Adrenal calcifications & ++ & $+1+++$ \\
Dyslipidemia & $5-160$ times higher than normal & +35 times higher than normal \\
Cholesteryl ester storage & In the liver: 2-10 times higher than normal & $1.5-3$ times higher than normal \\
Triglyceride storage & In the spleen: 8-100 times higher than normal & \\
& In the adrenal glands: 1.5-8 times higher than normal &
\end{tabular}

FIGURE 1. A. Effect of LAL-D on the liver and lipid metabolism. A hepatocyte receptor is responsible for LDL and VLDL cholesterol uptake, which are then carried to the lysosome. LAL-D results in cholesterol ester (CE) and triglyceride accumulation, and reduces free cholesterol (FC) and fatty acid (FA) production. The reduction in cholesterol increases sterol regulatory-element binding protein (SREBP) activity and cholesterol and FA synthesis, thus increasing LDL and VLDL cholesterol levels. The reduction in FC decreases liver X receptor (LXR) activation and the expression of ABCA1 transporter, thus reducing cholesterol flow to apolipoprotein A1 (ApoA-1) and plasma HDL cholesterol levels. B. Effect of treatment with sebelipase alfa (rhLAL); the mannose-6-phosphate (M6P) receptor is responsible for sebelipase alpha uptake, which is carried to the lysosome, where it reverses lipid metabolism defects. It prevents hepatocyte lipid accumulation. Modified from Rader D. ${ }^{24}$

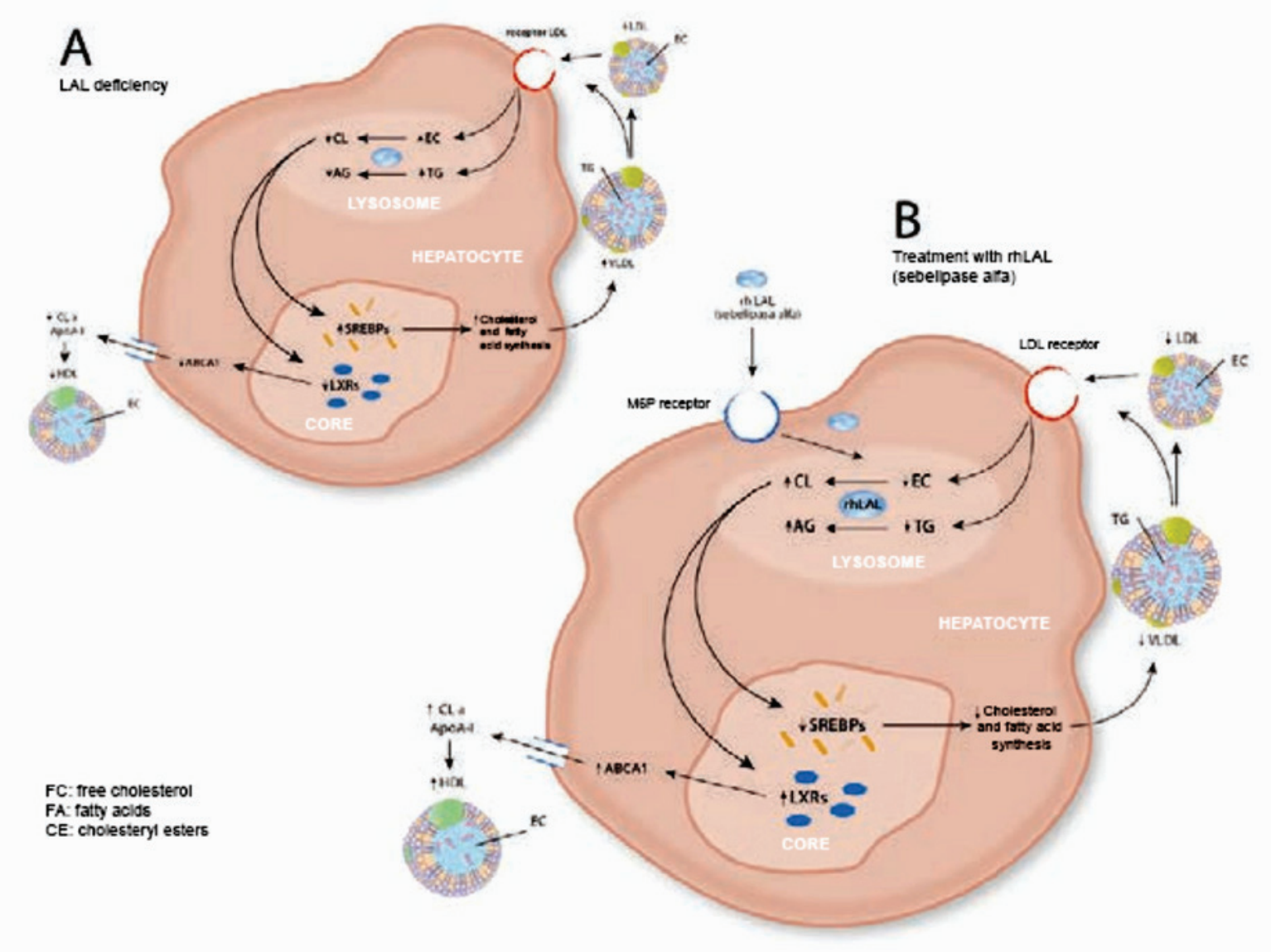

LAL: lysosomal acid lipase; HDL: high-density lipoproteins; LDL: low-density lipoproteins;

VLDL: very low density lipoproteins; TG: triglycerides. 
be diagnosed quickly, before any permanent damage occurs (in the liver, spleen, digestive tract, vascular system). ${ }^{11,12}$ Below we will review differential diagnoses.

Visceromegaly in the severe infant presentation or Wolman disease is also common in other storage diseases (Niemann-Pick disease, Gaucher disease, glycogen storage disease type IV). In the case of hemophagocytic lymphohistiocytosis, similarities have been described between this disease and LAL-D ${ }^{25}$ and other oncological diseases. Although these other conditions have their own typical phenotypes and biochemical features, the severe clinical form in the first year of life may be confused with acid lipase deficiency. ${ }^{26-31}$

Adrenal calcifications should be differentiated from neuroblastoma and adrenal hemorrhage, although in these cases, the lesion is usually unilateral.

Sepsis, congenital infections with visceromegaly, subacute or chronic gastroenteritis, malabsorption disorders, Hirschsprung disease, and cow's milk allergy may be confused with LAL-D, ${ }^{21}$ so it should be included in the differential diagnosis when any of these more common etiologies is not confirmed or if the patient's course does not improve as expected.

LAL-D should be included in the differential diagnosis of older patients (children, adolescents and adults) with normal weight or overweight, elevated levels of total cholesterol and LDL-C, low levels of HDL-C, elevated transaminases and/or hepatomegaly and/or hepatic steatosis. ${ }^{12}$

Overweight or obese patients with metabolic syndrome, ${ }^{32}$ elevated transaminases, signs of hepatic steatosis in imaging tests, and alcohol intake $<140 \mathrm{~g} /$ week, it is necessary to rule out other muscle or liver diseases besides nonalcoholic fatty liver disease (NAFLD)/ nonalcoholic steatohepatitis (NASH). ${ }^{32,33}$

Obesity itself does not warrant looking for NAFLD based on elevated transaminases, ${ }^{34}$ although LAL-D should be ruled out in those patients who lose weight on treatment but whose biochemical markers are not modified as expected..$^{25,35}$

As there is no specific biochemical marker for NAFLD and NASH, doing a liver biopsy has been the recommendation, ${ }^{36,37}$ although it does not provide a definitive diagnosis either. Patients with microvesicular steatosis may be wrongly diagnosed as having NAFLD ${ }^{35}$ or NASH. At present, NAFLD is the most common cause of elevated transaminases during childhood and adolescence, resulting from the increase in obesity incidence. ${ }^{38,39}$ Adolescents and adults with NAFLD usually have insulin resistance and hyperglycemia, but this is not the case in patients with LAL-D. ${ }^{40}$ NAFLD is rare among children younger than 3 years old, and uncommon among those younger than 10 years old. Table 2 describes the characteristics of NAFLD/NASH and LAL-D.

In Latin America, cirrhosis ${ }^{41}$ is associated with chronic alcohol abuse, hepatitis virus (hepatitis $\mathrm{C}$ virus [HCV]: 29\%; hepatitis B virus [HBV]: 27\%), alterations in liver drainage (19\%), NASH $(8 \%)$, and cryptogenic cirrhosis $(6 \%){ }^{42}$

In pediatrics, $5-15 \%$ of cirrhosis cases are cryptogenic, and LAL-D should be included

TABLE 2. Differential diagnosis of non-alcoholic fatty liver disease/non-alcoholic steatohepatitis and lysosomal acid lipase deficiency (late onset)

\begin{tabular}{lcc}
\hline & NAFLD/NASH & LAL-D (late onset) \\
\hline Prevalence & $20 / 100$ & $1: 40000$ \\
Hepatomegaly & $+/-$ & +++ \\
Splenomegaly & $+/-$ & ++ \\
BMI & Overweight/obesity & Normal/overweight \\
AST / ALT $\uparrow$ & $20 \%$ in the general population & +++ \\
Carbohydrate metabolism & Abnormal & Normal \\
Triglycerides & $\uparrow \uparrow$ & Normal or $\uparrow$ \\
HDL-C & $\downarrow$ & $\uparrow$ \\
LDL-C & Normal or $\uparrow$ & $\uparrow$ \\
Liver biopsy & Macrovesicular steatosis & Microvesicular steatosis \\
\hline
\end{tabular}

NAFLD/NASH: non-alcoholic fatty liver disease/non-alcoholic steatohepatitis; BMI: body mass index;

ALT: alanine aminotransferase; AST: aspartate aminotransferase; HDL-C: high-density lipoprotein cholesterol; LDL-C: low-density lipoprotein cholesterol. 
in screening tests for both children and adults. Microvesicular or mixed steatosis of the liver in children and adolescents leads to the suspicion of Wilson's disease, rather than LAL-D, because steatosis in Wilson's disease is secondary to copper accumulation, although LAL-D should also be included in the differential diagnosis. 43,35

In the case of early liver failure, LAL-D should be included in screening tests in addition to tyrosinemia, galactosemia, beta-oxidation disorders, neonatal hemochromatosis, and mitochondrial diseases. ${ }^{44,45}$

In the case of LAL-D, the histology of the liver shows cholesteryl ester accumulation in hepatocytes and Kupffer cells. Almost $50 \%$ of cases present with septal fibrosis that progresses to cirrhosis. A liver transplant may be required in the three years following symptom onset. ${ }^{6,11,12}$ The histopathological examination is facilitated by immunostaining of cathepsin $\mathrm{D}$, a lysosomal protein. ${ }^{11}$

In relation to dyslipidemia, there are national recommendations in place for its screening in children and adolescents aged 6 to $17 .{ }^{46,47}$ This allows for the detection of hypercholesterolemia and its early management. If there is no clear family history, and dyslipidemia does not improve with regular treatment (diet and physical activity to achieve a normal body mass index [BMI]), LAL-D should be investigated, as well as other causes of secondary hypercholesterolemia.

Pediatric patients with LDL $>130 \mathrm{mg} / \mathrm{dL}$ and / or $\mathrm{HDL}<40 \mathrm{mg} / \mathrm{dL}$ without a clear family history, obesity, or central fat distribution, or with normal fasting glucose may have LAL-D ${ }^{48}$ (Table 3 ). ${ }^{8}$

\section{TREATMENT}

No effective treatment for LAL-D has been established; lipid lowering drugs have been poorly effective and failed to improve liver involvement. ${ }^{1,11}$ There is scarce information about the history and follow-up of liver transplantation, although in the past it has been the only plausible option in the case of progression to end-stage liver failure. $^{1}$

In 2015, the use of an enzyme replacement therapy with a recombinant human form of the lysosomal enzyme (sebelipase) was approved for intravenous use every 15 days. A doubleblind, randomized, multicenter clinical trial, ${ }^{49}$ demonstrated a significant decrease in transaminases, liver fat as observed in a magnetic resonance imaging (MRI) study, and hepatomegaly. LDL-C and triglycerides were significantly reduced, and HDL-C increased. Only a few, mild adverse reactions were reported in this study (Figure 1. B).

In a phase $2 / 3$ clinical trial with sebelipase alfa in children with the early onset subtype, 5 out of 9 patients survived beyond 2 years old, and 1 , beyond 5 years old. Liver disease markers, gastrointestinal symptoms, and nutritional status improved. ${ }^{50}$

Although no long-term follow-up has been completed for this treatment, given the mechanism of action of sebelipase, it may have a potential effect on the reduction of liver fibrosis and its progression to cirrhosis, as well as on the improvement of dyslipidemia and the prevention of cardiovascular complications. ${ }^{51}$

TABLE 3. High-risk populations that should be considered in the screening for lysosomal acid lipase deficiency

Modified from Burton B, JPGN 2015;61:619-625. ${ }^{8}$

Non obese patients with the following:

- Persistent hepatomegaly

- Elevated transaminases without a definite cause

- High levels of LDL (> $130 \mathrm{mg} / \mathrm{dL}, 4.1 \mathrm{mmol} / \mathrm{L}$ )

- Low levels of HDL (males: $<40 \mathrm{mg} / \mathrm{dL}$; females: $<50 \mathrm{mg} / \mathrm{dL}$ )

Obese patients diagnosed with metabolic syndrome and the following:

- Persistent hepatomegaly

- No hyperglycemia

- Elevated transaminases without a definite cause that do not improve with a reduction in BMI

Obese patients who manage to reduce their BMI but whose lipid profile does not improve Patients with microvesicular steatosis

HDL: high-density lipoproteins; LDL: low-density lipoproteins; BMI: body mass index. 


\section{REFERENCES}

1. Reiner Z, Guardamagna O, Nair D, Soran H, et al. Lysosomal acid lipase deficiency an under-recognized cause of dyslipidaemia and liver dysfunction. Atherosclerosis 2014;235(1):21-30.

2. Grabowski G, Charnas L, Du H. Lysosomal Acid Lipase Deficiencies: The Wolman Disease/Cholesteryl Ester Storage Disease Spectrum. In Valle D, Beaudet AL, Vogelstein B, eds. The Online Metabolic and Molecular Bases of inherited Diseases. New York: McGraw-Hill, 2013.

3. KonnoT,Fujii M, WatanukiT,KoizumiK.Wolman's disease: the first case in Japan. Tohoku J Exp Med 1966;90(4):375-89.

4. Crocker AC, Vawter GF, Neuhauser EB, Rosowsky A. Wolman's disease: three new patients with a recently described lipidosis. Pediatrics 1965;35:627-40.

5. Gómez-Nájera M, Barajas-Medina H, Gallegos-Rivas M, Mendez-Sashida P, et al. New Diagnostic Method for Lysosomal Acid Lipase Deficiency and the Need to recognize its Manifestation in infants (Wolman Disease). J Pediatr Gastroenterol Nutr 2015;60(3):e22-4.

6. Abramov A, Schorr S, Wolman M. Generalized xanthomatosis with calcified adrenals. AMA J Dis Child 1956;91(3):282-6.

7. Amitai N, Grozovski S, Landau D. Adrenal calcification in an infant with cholestasis. J Pediatr 2015;167(5):1163.

8. Burton BK, Deegan PB, Enns GM, Guardamagna O, et al. Clinical Features of Lysosomal Acid Lipase Deficiency. J Pediatr Gastroenterol Nutr 2015;61(6):619-25.

9. BernsteinDL, Hülkova H, Bialer MG, Denick RJ. Cholesteryl ester storage disease: review of the findings in 135 reported patients with an underdiagnosed disease. $J$ Hepatol 2013;58(6):1230-43.

10. Anderson RA, Bryson GM, Parks JS. Lysosomal acid lipase mutations that determine phenotype in Wolman and cholesterol ester storage disease. Mol Genet Metab 1999;68(3):333-45.

11. Marshall WC, Ockenden BG, Fosbrooke AS, Cumings JN. Wolman's disease. A rare lipidosis with adrenal calcification. Arch Dis Child 1969;44(235):331-41.

12. Mayatepek E, Seedorf U, Wiebusch H, Lenhartz H, et al. Fatal genetic defect causing Wolman disease.J Inherit Metab Dis 1999;22(1):93-4.

13. Sjouke B, Van der Stappen JW, Groener JE, Pepping A, et al. Hypercholesterolaemia and hepatosplenomegaly: Two manifestations of cholesteryl ester storage disease. Neth J Med 2015;73(3):129-32.

14. Decarlis S, Agostoni C, FerranteF, ScarlinoS, etal.Combined hyperlipidaemia as a presenting sign of cholesteryl ester storage disease. J Inherit Metab Dis 2009;32(Suppl 1):S11-3.

15. Dalg1ç B, Sarı S, Gündüz M, Ezgü F, et al. Cholesteryl ester storage disease in a young child presenting as isolated hepatomegaly treated with simvastatin. Turk J Pediatr 2006;48(2):148-51.

16. Haller W, Sharif K, Millar A, Brown R, et al. Gallbladder Dysfunction in Cholesterol Ester Storage Disease. J Pediatr Gastroenterol Nutr 2010;50(5):555-8.

17. Stenson PD, Mort M, Ball EV, Howells K, et al. The Human gene mutation database: 2008 update. Genome Med 2009;22(1):13

18. Klima H, Aslanidis C, Fehringer P, Lackner KJ, et al. A splice junction mutation causes deletion of a 72-base exon from the mRNA for lysosomal acid lipase in a patient with cholesteryl ester storage disease. J Clin Invest 1993;92(6):2713-8.

19. Scott SA, Liu B, Nazarenko I, Martis S, et al. Frequency of the cholesteryl ester storage disease common LIPA E8SJM mutation (c.894G $>$ A) in various racial and ethnic groups. Hepatology 2013;58(3):958-65.

20. Muntoni S, Wiebusch H, Jansen-Rust M, Rust S, et al.
Prevalence of cholesteryl ester storage disease. Arterioscler Thromb Vasc Biol 2007;27(8):1866-8.

21. Guy GJ, Butterworth J. Acid esterase activity in cultured skin fibroblasts and amniotic fluid cells using 4-methylumbelliferyl palmitate. Clin Chim Acta 1978;84(3):361-71.

22. Hamilton J, Jones I, Srivastava R, Galloway P. New method for the measurement of lysosomal acid lipase in dried blood spots using the inhibitor Lalistat 2. Clin Chim Acta 2012;413(15-16):1207-10.

23. Fasano T, Pisciotta L, Bocchi L, Guardamagna O, et al. Lysosomal lipase deficiency: molecular characterization of eleven patients with Wolman or cholesteryl ester storage disease. Mol Genet Metab 2012;105(3):450-6.

24. Rader D. Lysosomal Acid Lipase Deficiency- A new therapy for a genetic Lipid disease. N Engl J Med 2015; 373(11): 1071-3.

25. Rabah F, Al-Hashmi N, Beshlawi I. Wolman's Disease with secondary Hemophagocytic Lymphohistiocytosis. Pediatr Hematol Oncol 2014;31(6):576-8.

26. HoffmanEP, BarrML, Giovanni MA, Murray M. Lysosomal Acid Lipase Deficiency. In: Pagon RA, Adam MP, Ardinger $\mathrm{HH}$, et al, eds. GeneReviews ${ }^{\circledR}$ [Internet]. Seattle (WA): University of Washington; 2016. [Accessed on: November $\left.30^{\text {th }}, 2016\right]$. Available at: http:/ / www.ncbi.nlm.nih.gov/ books/NBK305870/.

27. MosesSW, Parvari R. The variable presentation of glycogen storage disease type IV: a review of clinical enzymatic and molecular studies. Curr Mol Med 2002;2(2):178-88.

28. MignotC, Gelot A, Bessières B, Daffos F, etal. Perinatal-lethal Gaucher disease. Am J Med Genet A 2003;120A(3):338-44.

29. Grabowsky GA, Andria G, Baldellou A, et al. Pediatric non-neuropathic Gaucher disease: presentation, diagnosis and assessment. Consensus statment. Eur J Pediatr 2004;163(2):58-66.

30. Vanier MT, Millat G. Niemann Pick disease type C. Clin Genet 2003;64(4):269-81.

31. Niemann-Pick disease. In Nyhan W, Barshop B, Al-Apeel A. Atlas of Inherited Metabolic Diseases. 3rd ed. London: Hodder Arnold; 2012:708-17.

32. Grundy SM, Brewer HB Jr, Cleeman JI, Smith SC Jr, et al. Definition of metabolic syndrome: Report of the National Heart, Lung, and Blood Institute/American Heart Association conference on scientific issues related to definition. Circulation 2004;109(3):433-8.

33. WGO Global Guidelines. Non-alcoholic fatty liver disease and nonalcoholic steatohepatits. 2012. [Accessed on: November $\left.30^{\text {th }}, 2016\right]$. Available at: http://www. worldgastroenterology.org/UserFiles / file/guidelines / nafld-nash-english-2012.pdf.

34. Vajro P, Lenta S, Socha P, Dhawan A, et al. Diagnosis of nonalcoholic fatty liver disease in children and adolescent: position paper of the ESPGHAN. Hepatology Committee. J Pediatr Gastroenterol Nutr 2012;54(5):700-13.

35. Chalasani N, Younossi Z, Lavine JE, Diehi AM, et al. The diagnosis and management of non-alcoholic fatty liver disease: Practice Guideline by the American Association for the Study of Liver Diseases, American College of Gastroenterology, and the American Gastroenterological Association. Hepatology 2012;55(6):2005-23.

36. Neuschwander-Tetri BA, Caldwell SH. Nonalcoholic steatohepatitis: Summary of an AASLD Single Topic Conference. Hepatology 2003;37(5):1202-19.

37. Brunt EM, Kleiner DE, Wilson LA, Belt P, et al. Nonalcoholic fatty liver disease (NAFLD) activity score and the histopathologic diagnosis in NAFLD: distinct clinicopathologic meanings. Hepatology 2011;53(3):810-20.

38. Santos LF, Hernández G, Varón Puerta A, Beltrán O, et al. 
Enfermedad hepática por infiltración grasa no alcohólica. La nueva pandemia del milenio. Rev Col Gastroenterol 2010;25(4):380-98.

39. WHO. Report of the commission on ending childhood obesity. Ginebra: WHO; 2016. [Accessed on: November $\left.30^{\text {th }}, 2016\right]$. Available at: http://apps.who.int/iris/ bitstream/10665/204176/1/9789241510066_eng.pdf.

40. Semple RK, Sleigh A, Murgatroyd PR, Adams CA, et al. Postreceptor insulin resistance contributes to human dyslipidemia and hepatic steatosis. J Clin Invest 2009;119(2):315-22.

41. McCormick PA. Hepatic Cirrhosis. In: Dooley JA, Lok ASF, Burton AK, Heathcote EJ, eds. Sherlock's Diseases of the Liver and Biliary System. 12th ed. Oxford: WilleyBlackwell; 2011:103-20.

42. Piñero F, Tisi Baña M, de Ataide EC, Hoyos Duque S, et al. Liver Transplantation for Hepatocellular carcinoma: Evaluation of the alfa-fetoprotein model in a multicenter cohort from Latin America. Liver Int 2016;36(11):1657-67.

43. Rodríguez-Castro KI, Hevia-Urrutia FJ, Sturniolo GC. Wilson's disease: A review of what we have learned. World J Hepatol 2015;7(29):2859-70.

44. Molleston JP, Sokol RJ, Karnsakul W, Miethke A et al. Evaluation of the Child With Suspected Mitochondrial Liver Disease. J Pediatr Gastroenterol Nutr 2013;57(3):269-76.
45. Bliksrud Y, Ellingsen A, Bjørås M. Fumarylacetoacetate inhibits the initial step of the base excision repair pathway: implication for the pathogenesis of tyrosinemia type I. J Inherit Metab Dis 2013;36(5):773-8.

46. Expert Panel on Integrated Guidelines for Cardiovascular Health and Risk Reduction in Children and Adolescents: Summary Report. Pediatrics 2011;128(Suppl 5):S213-56.

47. Comité De Nutrición. Consenso sobre Manejo de las Dislipidemias en Pediatría. Arch Argent Pediatr 2015;113(2):177-86.

48. Alberti K, Zimmet P, Shaw J. Metabolic syndrome-a new world-wide definition. A Consensus Statement from the International Diabetes Federation. Diabet Med 2006;23(5):469-80.

49. Burton BK, Balwani M, FeilletF, BarićI, et al. A Phase 3 Trial of Sebelipase Alfa in Lysosomal Acid Lipase Deficiency. N Engl J Med 2015;373(11):1010-20.

50. Valayannopoulos V, Plantaz D, Vara R. Eckert S, et al. Clinical effect of sebelipase alfa on survival and growth in infants with lysosomal acid lipase deficiency (Wolmam Disease). Mol Genet Metab 2014,111(2):S108.

51. Paton DM. Sebelipase alfa: enzymatic replacement treatment for lysosomal acid lipase deficiency. Drugs Today (Barc) 2016:52(5):287-93. 